\title{
Non-equilibrium Chemistry in the Atmospheres of Brown Dwarfs
}

\section{Saumon}

Department of Physics and Astronomy, Vanderbilt University, Nashville, TN 37235

\author{
M. S. Marley
}

NASA Ames Research Center, Moffett Field, CA 94035

K. Lodders

Dept. of Earth \& Planetary Science, Washington University, St-Louis, MO 63130-4899

\section{R. S. Freedman}

The Space Physics Research Institute, NASA Ames Research Center, Moffett Field, CA 94035

\begin{abstract}
Carbon monoxide and ammonia have been detected in the spectrum of Gl $229 \mathrm{~B}$ at abundances that differ substantially from those obtained from chemical equilibrium. Vertical mixing in the atmosphere is a mechanism that can drive slowly reacting species out of chemical equilibrium. We explore the effects of vertical mixing as a function of mixing efficiency and effective temperature on the chemical abundances in the atmospheres of brown dwarfs and on their spectra. The models compare favorably with the observational evidence and indicate that vertical mixing plays an important role in brown dwarf atmospheres.
\end{abstract}

\section{Introduction}

The discovery of strong methane bands in the spectrum of Gl 229B indicated at once that it has a very low effective temperature $(\sim 1000 \mathrm{~K})$ and it was hailed as the first clearly identified brown dwarf (Nakajima et al. 1995; Oppenheimer et al. 1995). This follows from the chemistry of carbon in stellar atmospheres. In cool $\mathrm{M}$ dwarfs, carbon is predominantly in the form of carbon monoxide (CO), while in Jovian planets methane $\left(\mathrm{CH}_{4}\right)$ is the dominant form. The transition occurs in the brown dwarf regime, at $T \sim 1100 \mathrm{~K}$ for a pressure of 1 bar (Fegley \& Lodders 1996).

The presence of methane bands is so striking that it prompted the creation of the new spectral class of $T$ dwarfs (Kirkpatrick et al. 1999). Gl 229B is a T6 dwarf. In this context, the discovery of a strong CO band in the $4.5-5 \mu \mathrm{m}$ spectrum of Gl 229B by Noll, Geballe \& Marley (1997) is quite remarkable. Analysis of the spectrum reveals an abundance of $\mathrm{CO}$ that is over 3 orders of 
magnitude larger than expected from chemical equilibrium calculations (Noll et al. 1997; Griffith \& Yelle 1999; Saumon et al. 2000), as anticipated by Fegley \& Lodders (1996). A similar situation is observed in the atmosphere of Jupiter where $\mathrm{CO}$ is detected while chemical equilibrium overwhelmingly favors $\mathrm{CH}_{4}$ as the reservoir of carbon (Prinn \& Barshay 1977).

The excess of CO in Gl 229B can be understood if the chemistry of carbon is driven away from equilibrium by vertical mixing on a time scale shorter than the rate of conversion of $\mathrm{CO}$ into $\mathrm{CH}_{4}$ (Fegley \& Lodders 1996; Noll et al 1997; Griffith \& Yelle 1999).

The two reservoirs of nitrogen in brown dwarfs are molecular nitrogen $\left(\mathrm{N}_{2}\right)$ and ammonia $\left(\mathrm{NH}_{3}\right)$. Ammonia is the dominant molecule at low temperatures. While $\mathrm{N}_{2}$ is invisible in the near infrared, Saumon et al. (2000) detected $\mathrm{NH}_{3}$ features in Gl 229B in the $K$ band and inferred its presence in the $H$ band. Each detection corresponds to a different level in the atmosphere. $\mathrm{NH}_{3}$ abundances determined separately for each band reveal a value in agreement with chemical equilibrium in the $H$ band and a depletion by at least a factor of 4 in the $K$ band. These results can be interpreted consistently with the $\mathrm{CO}$ observation by invoking vertical mixing in the atmosphere on a time scale shorter than the rate of conversion of $\mathrm{N}_{2}$ into $\mathrm{NH}_{3}$.

The modeling of non-equilibrium chemistry of $\mathrm{CO}$ and other molecules due to mixing in planetary atmospheres is a mature field (e.g. Barshay \& Lewis 1978; Fegley \& Prinn 1985; Fegley \& Lodders 1994; Lodders \& Fegley 1994). Application to brown dwarfs have so far been limited to modeling the excess of $\mathrm{CO}$ in Gl 229B (Griffith \& Yelle 1999) and a detailed study of the kinetics of CNO chemistry (Lodders \& Fegley 2002). Here we present the first systematic exploration of the effects of mixing on the chemistry of carbon and nitrogen in brown dwarfs atmospheres. We first summarize the main features of nonequilibrium chemistry of carbon and nitrogen caused by vertical mixing. Using a simple parametrization for the rate of mixing, we then compute the resulting non-equilibrium abundances of $\mathrm{CO}, \mathrm{CH}_{4}, \mathrm{~N}_{2}, \mathrm{NH}_{3}$ and $\mathrm{H}_{2} \mathrm{O}$ as a function of effective temperature and mixing efficiency. The new abundance profiles lead to significant changes in the emergent spectra of cool brown dwarfs.

\section{Carbon and Nitrogen Chemistry}

The relative abundances of $\mathrm{CO}$ and $\mathrm{CH}_{4}$ can be driven out of equilibrium in the cool atmospheres of brown dwarfs and giant planets because of the strong asymmetry in the reaction rates of the (net) reaction

$$
\mathrm{CO}+3 \mathrm{H}_{2} \leftrightarrow \mathrm{CH}_{4}+\mathrm{H}_{2} \mathrm{O} .
$$

Because of the large binding energy of $\mathrm{CO}$, the reaction proceeds much more slowly to the right than to the left. Vertical mixing dredges up hot gas that is relatively rich in $\mathrm{CO}$ to the cooler part of the atmosphere, where $\mathrm{CO}$ is converted very slowly into $\mathrm{CH}_{4}$, while the $\mathrm{CH}_{4}$ that is brought down is quickly converted into $\mathrm{CO}$. The net result is an overabundance of $\mathrm{CO}$, with the $\mathrm{CO} / \mathrm{CH}_{4}$ ratio in the upper atmosphere fixed to its value at the level where the mixing time scale and the chemical time scale are equal. Nearly all of the elemental oxygen 
is partitioned between condensates, carbon monoxide and water. It follows that an overabundance of $\mathrm{CO}$ implies an underabundance of $\mathrm{H}_{2} \mathrm{O}$.

The conversion of $\mathrm{N}_{2}$ (favored at higher temperatures) into $\mathrm{NH}_{3}$ is entirely analogous to that of $\mathrm{CO} / \mathrm{CH}_{4}$. The net reaction is

$$
\mathrm{N}_{2}+3 \mathrm{H}_{2} \leftrightarrow 2 \mathrm{NH}_{3} .
$$

The large binding energy of $\mathrm{N}_{2}$ leads to a slow reaction rate to the right, while the reaction to the left proceeds rapidly. Thermochemical and kinetic data indicate that all other molecular and atomic species identified in the spectra of brown dwarfs react on very short time scales and are expected to be in chemical equilibrium. In brown dwarfs, non-equilibrium chemistry induced by vertical mixing is thus limited to the five species $\mathrm{CO}, \mathrm{CH}_{4}, \mathrm{H}_{2} \mathrm{O}, \mathrm{NH}_{3}$ and $\mathrm{N}_{2}$.

The correct chemical pathway and reaction time scale for the conversion of $\mathrm{CO}$ into $\mathrm{CH}_{4}$ remains somewhat uncertain (Griffth \& Yelle 1999; Lodders \& Fegley 2002). For the purposes of this exploratory calculation, however, this is not a critical consideration and we adopt the chemical time scales for the net reactions (1) and (2) given in Lodders \& Fegley (2002).

\section{Vertical Transport}

In radiative atmospheres, vertical mixing can occur by eddy turbulence. This process is analogous to diffusion and occurs over a characteristic time scale

$$
\tau_{\text {mix }} \sim \frac{H^{2}}{K}
$$

where $H$ is the pressure scale height and $K$ is the coefficient of diffusion. While $K$ is a free parameter in our calculation, it ranges from $\sim 10^{2}$ to $10^{5} \mathrm{~cm}^{2} / \mathrm{s}$ in planetary stratospheres.

Atmospheres of brown dwarfs all become convective below a certain depth and convection is a very effective mixing mechanism. The time scale for convective mixing is

$$
\tau_{\text {mix }}=\tau_{\text {conv }} \sim \frac{H_{c}}{v_{c}}=\frac{H^{2}}{K_{c}},
$$

where $H_{c}$ and $v_{c}$ are the convective mixing length and velocity, respectively. For convenience, we choose $H_{c}=H$. An effective diffusion coefficient, $K_{c}$, can be defined for convective mixing (Eq. 4) and evaluated using the mixing length formalism. In brown dwarf atmospheres, we find $K_{c} \sim 10^{8}-10^{9} \mathrm{~cm}^{2} / \mathrm{s}$. Thus, the transition from a convective zone to a radiative zone in the atmosphere is accompanied by a sudden decrease in the efficiency of mixing.

\section{Non-Equilibrium Chemistry in Brown Dwarf Atmospheres}

We model the effects of vertical transport on the chemistry of carbon and nitrogen in cloudless atmospheres with $700 \leq T_{\text {eff }} \leq 2000 \mathrm{~K}, g=10^{5} \mathrm{~cm} / \mathrm{s}^{2}$, and solar abundances. The eddy diffusion coefficient in the radiative zone is varied 


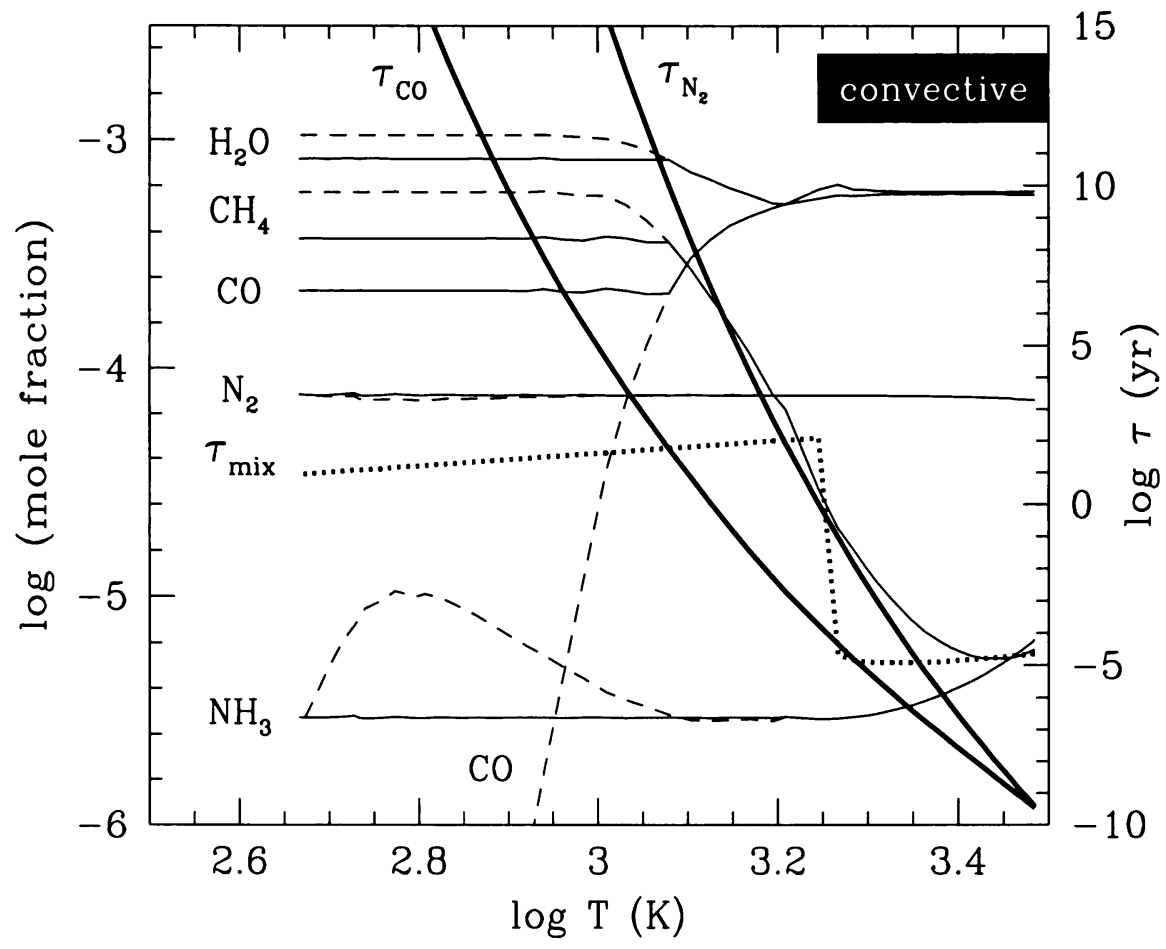

Figure 1. Chemistry inside a $T_{\text {eff }}=1200 \mathrm{~K}, g=10^{5} \mathrm{~cm} / \mathrm{s}^{2}$ cloudless atmosphere. Dashed lines show the equilibrium abundances of molecules of interest and solid lines show the abundances that result from non-equilibrium chemistry driven by vertical transport. In the radiative zone, the eddy diffusion coefficient is set to $K=100 \mathrm{~cm}^{2} / \mathrm{s}$. The extent of the convection zone is indicated in the upper right corner.

from $K=10^{2}$ to $10^{10} \mathrm{~cm}^{2} / \mathrm{s}$ while in the convective zone the mixing time scale is calculated from the mixing length formalism.

An example of the effect of vertical mixing on the chemical abundance profiles in a model atmosphere is shown in Figure 1. Depth in the atmosphere is indicated by the local temperature. The equilibrium abundances, shown with dashed lines, clearly show the transition in carbon chemistry from CO deep in the atmosphere to $\mathrm{CH}_{4}$ near the surface. The oxygen freed by the conversion of $\mathrm{CO}$ into $\mathrm{CH}_{4}$ toward the top of the atmosphere is used to form $\mathrm{H}_{2} \mathrm{O}$. The $\mathrm{H}_{2} \mathrm{O}$ abundance thus increases in concert with that of $\mathrm{CH}_{4}$. In this relatively hot model, nearly all nitrogen is in the form of $\mathrm{N}_{2}$ and the $\mathrm{NH}_{3}$ abundance remains very low throughout the atmosphere.

The chemical time scales for the conversion of $\mathrm{CO}$ and $\mathrm{N}_{2}$ are shown by the two heavy solid lines. These vary by more than 25 orders of magnitude throughout the atmosphere! Mixing occurs rapidly in the convection zone but is considerably less efficient in the radiative zone for our choice of $K=100 \mathrm{~cm}^{2} / \mathrm{s}$. In regions where $\tau_{\text {chem }}<\tau_{\text {mix }}$, chemical equilibrium prevails. Where $\tau_{\text {chem }}>\tau_{\text {mix }}$, 


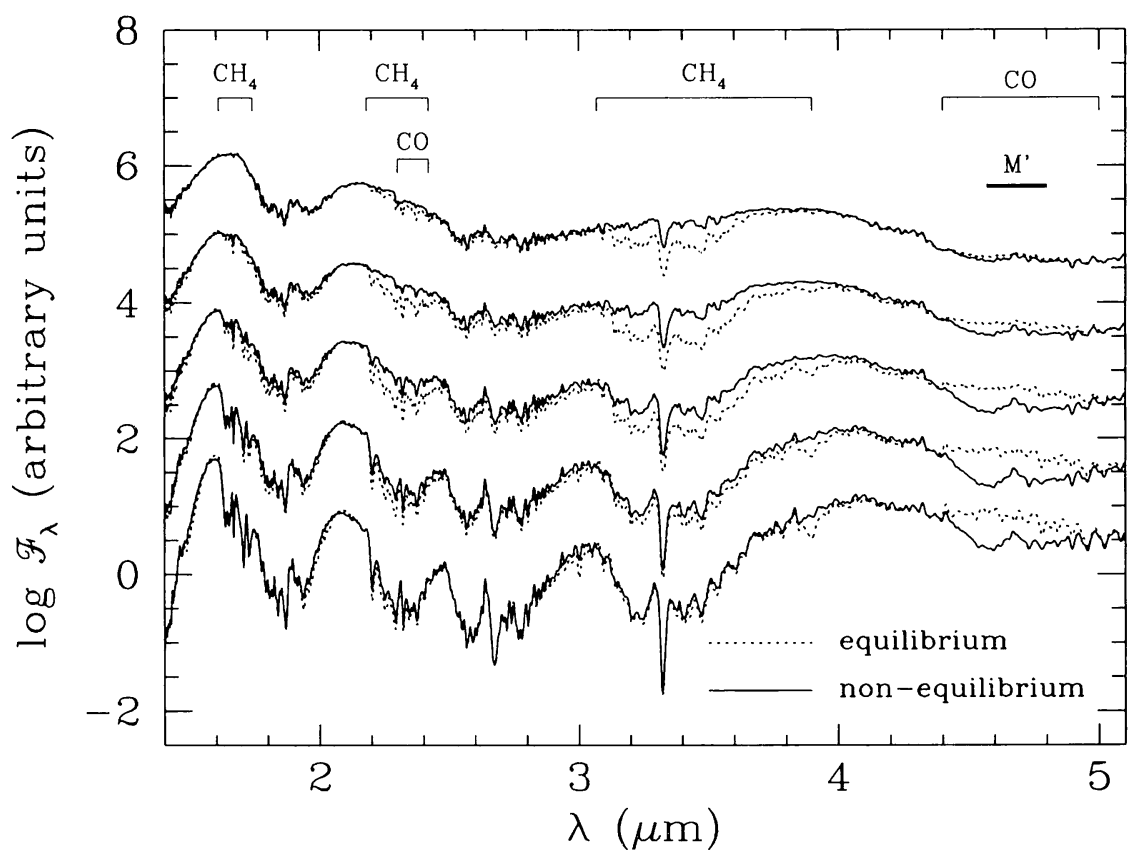

Figure 2. A comparison of spectra computed with abundances from chemical equilibrium and with the non-equilibrium abundances resulting from vertical transport. All models have $g=10^{5} \mathrm{~cm} / \mathrm{s}^{2}$ and $K=10^{4} \mathrm{~cm}^{2} / \mathrm{s}$. $T_{\text {eff }}$ decreases from $1600 \mathrm{~K}$ (top) to $800 \mathrm{~K}$ (bottom) in steps of $200 \mathrm{~K}$. The spectra are plotted at a resolution of $R=200$ and are offset vertically for clarity. The main bands of $\mathrm{CO}$ and $\mathrm{CH}_{4}$ and the bandpass of the $M^{\prime}$ filter are indicated.

however, the mole fractions are determined by their values at the crossing point. As can be seen in Fig. 1, there can be more than one level where $\tau_{\text {chem }}=\tau_{\text {mix }}$, resulting in alternating zones in and out of chemical equilibrium. For simplicity, we consider only the uppermost (lowest $\mathrm{T}$ ) crossing. It turns out that in all cases, this is an excellent approximation to the more complex, full solution because the species affected have either 1) very low abundances or 2) have nearly flat abundance profiles where the deeper crossings occur and are unaffected by mixing.

The resulting non-equilibrium abundances are shown by solid lines in Fig. 1. The abundance of $\mathrm{CO}$ is considerably enhanced by vertical transport while both $\mathrm{CH}_{4}$ and $\mathrm{H}_{2} \mathrm{O}$ are reduced by more modest factors. $\mathrm{N}_{2}$ is "frozen" at a deeper level than $\mathrm{CO}$. Its abundance is barely affected in this model, while the $\mathrm{NH}_{3}$ abundance decreases. The net effect of vertical transport in all models is an increase in the abundances of $\mathrm{CO}$ and $\mathrm{N}_{2}$, and a decrease in the abundances of $\mathrm{CH}_{4}, \mathrm{H}_{2} \mathrm{O}$ and $\mathrm{NH}_{3}$. Shortening the mixing time scale in the radiative zone (i.e. increasing $K$ ) produces larger changes in the abundances for fixed $T_{\text {eff }}$ and gravity. 


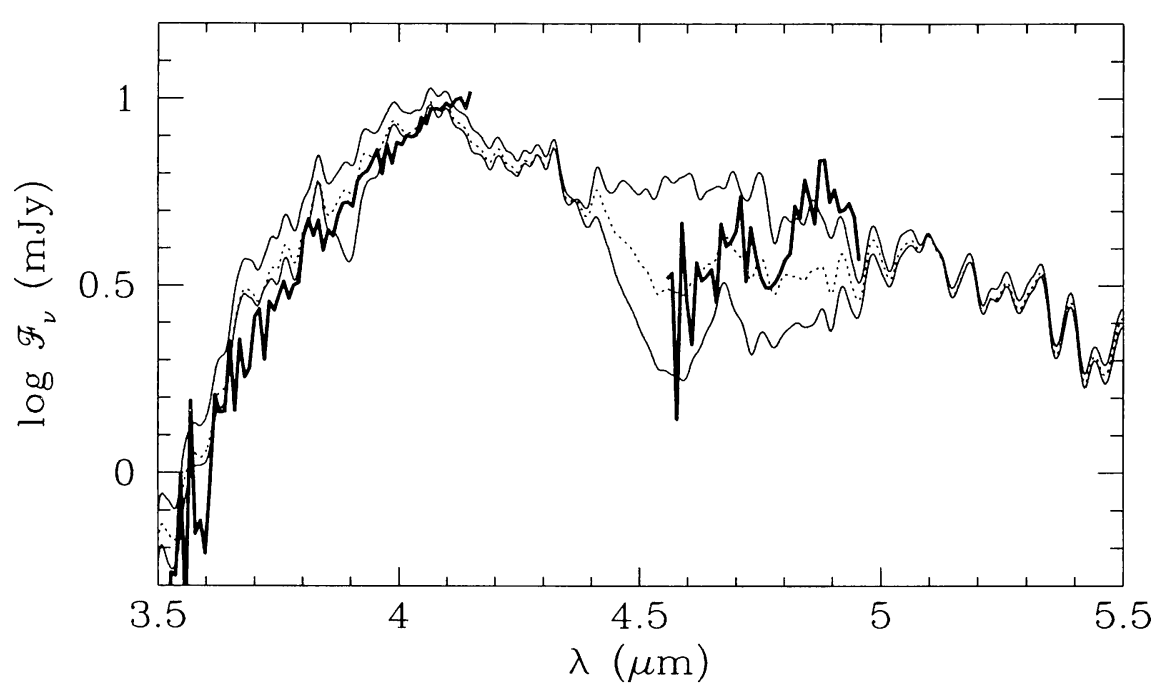

Figure 3. Carbon monoxide in Gl 229B. The spectrum of Gl 229B is shown as a thick solid line (Oppenheimer et al. 1998; Leggett et al. 1999). The $4.5-5 \mu \mathrm{m}$ spectrum is flux-calibrated using the $M^{\prime}$ photometry of Golimowski et al. (2002). Thin curves are models with $T_{\text {eff }}=1000 \mathrm{~K}, g=10^{5} \mathrm{~cm} / \mathrm{s}^{2}$ and $K=0,10^{2}$ and $10^{4} \mathrm{~cm}^{2} / \mathrm{s}$, from top to bottom, respectively. $K=0$ corresponds to the chemical equilibrium case. The absolute flux level of the models has not been adjusted to fit the data. The models are plotted at a resolution of $R=200$.

The effect of non-equilibrium chemistry on the spectra of brown dwarfs is shown in Figure 2. The change in the strength of the $4.7 \mu \mathrm{m}$ band of $\mathrm{CO}$ increases gradually toward lower $T_{\text {eff }}$ up to a maximum at $T_{\text {eff }} \sim 900 \mathrm{~K}$. The corresponding depletion in $\mathrm{CH}_{4}$ results in weaker $\mathrm{CH}_{4}$ bands with a maximum effect at $T_{\text {eff }} \sim 1400 \mathrm{~K}$. The $3.3 \mu \mathrm{m}$ band of $\mathrm{CH}_{4}$ is the most strongly affected, followed by the $2.2 \mu \mathrm{m}$ and $1.6 \mu \mathrm{m}$ bands.

The strongest features of $\mathrm{NH}_{3}$ appear in the $10-11 \mu \mathrm{m}$ region and have not yet been observed in any brown dwarf. Ammonia forms only at relatively low temperatures $\left(T_{\text {eff }} \lesssim 1200 \mathrm{~K}\right)$ and the strength of the features increases monotonically with decreasing $T_{\text {eff }}$. Vertical mixing systematically reduces the abundance of $\mathrm{NH}_{3}$ compared to its equilibrium value. Since $\mathrm{NH}_{3}$ is detectable at three different levels in the atmosphere (in the $N, K$, and $H$ bands; Saumon et al. 2000), the flat abundance profile predicted by this model (Fig. 1) can in principle be tested observationally.

Saumon et al. (2000) argued that the most reliable diagnostic of metallicity for $\mathrm{T}$ dwarfs at present is the forest of $\mathrm{H}_{2} \mathrm{O}$ lines in regions of the spectrum where water is the dominant absorber. A determination of the metallicity based on $\mathrm{H}_{2} \mathrm{O}$ lines would be affected by non-equilibrium chemistry, which can reduce its abundance by up to $\sim 0.3$ dex (increasing with $K$ and $T_{\text {eff }}$ ). 


\section{Observational Evidence}

Gl $229 \mathrm{~B}$ is the only $\mathrm{T}$ dwarf with a published $4.5-5 \mu \mathrm{m}$ spectrum (Noll et al. 1997; Oppenheimer et al. 1998) but $M^{\prime}$ band photometry is now available for several other brown dwarfs (Leggett et al. 2002; Golimowski et al. 2002). The $M^{\prime}$ band is a very good probe of the strength of the CO band (Fig. 2). Non-equilibrium spectra are compared with the spectrum of Gl 229B in Figure 3. A fair agreement with the rather noisy $4.5-5 \mu \mathrm{m}$ spectrum is obtained for $K \sim 10^{2} \mathrm{~cm}^{2} / \mathrm{s}$.

Absolute $M^{\prime}$ photometry is shown in Figure 4. The equilibrium models (dashed curve) overestimate the $M^{\prime}$ flux at lower $T_{\text {eff }}$. Non-equilibrium models can reproduce the observed fluxes quite well with $K \sim 10^{2}-10^{4} \mathrm{~cm}^{2} / \mathrm{s}$. Figure 4 suggests that increased $\mathrm{CO}$ absorption resulting from vertical transport is not a peculiarity of Gl $229 \mathrm{~B}$ but a feature common to late $\mathrm{L}$ dwarfs and $\mathrm{T}$ dwarfs.

\section{Conclusions}

Motivated by observations in the T6 dwarf Gl 229B of a strong CO band where none was expected and of a depletion of $\mathrm{NH}_{3}$, we have explored the effects of vertical mixing in brown dwarf atmospheres on the most prominent molecules visible in their spectra. Vertical mixing, by convection or eddy turbulence, can occur fast enough to keep abundances of $\mathrm{CO}, \mathrm{CH}_{4}, \mathrm{H}_{2} \mathrm{O}$ and $\mathrm{NH}_{3}$ from reaching their values at chemical equilibrium. Increased efficiency of mixing leads to an enrichment of $\mathrm{CO}$ and a depletion of $\mathrm{CH}_{4}, \mathrm{H}_{2} \mathrm{O}$, and $\mathrm{NH}_{3}$. The model of vertical mixing can explain the $\mathrm{CO}$ and $\mathrm{NH}_{3}$ observations in $\mathrm{Gl} 229 \mathrm{~B}$ selfconsistently. Furthermore, observations of $\mathrm{NH}_{3}$ in three different bandpasses can test the model prediction of a flat abundance profile throughout the atmosphere (Saumon et al. 2002). In this study, the efficiency of mixing is treated as a free parameter but it can eventually be calibrated with data.

New observational evidence suggests that the overabundance of $\mathrm{CO}$ observed in Gl 229B may be a common feature of late L dwarfs and of T dwarfs. If this is indeed the case, it becomes essential to include non-equilibrium chemistry in modeling the atmospheres and spectra of cool brown dwarfs. Because it affects the relative abundances of major constituents of the atmosphere, vertical mixing further complicates the determination of the composition of brown dwarfs.

Finally, vertical mixing can result in a suppression of the $M^{\prime}$ flux by up to one magnitude for $T_{\text {eff }}$ between $\sim 700$ and $1100 \mathrm{~K}$ (Fig. 4). This must be taken into account in plans to image extrasolar giant planets by capitalizing on their predicted superthermal $M$ band flux (Marley et al. 1996, Burrows et al. 1997).

Acknowledgments. We thank S. K. Leggett and D. A. Golimowski for kindly providing data in advance of publication. This work was supported in part by NSF grant AST-0086288 and NASA grant NAG5-9273 to M. S. M. and grant NAG5-4970 to R. S. F. Work of K. L. is supported by NASA grant NAG5-11958 and NSF grant AST-0086487. 


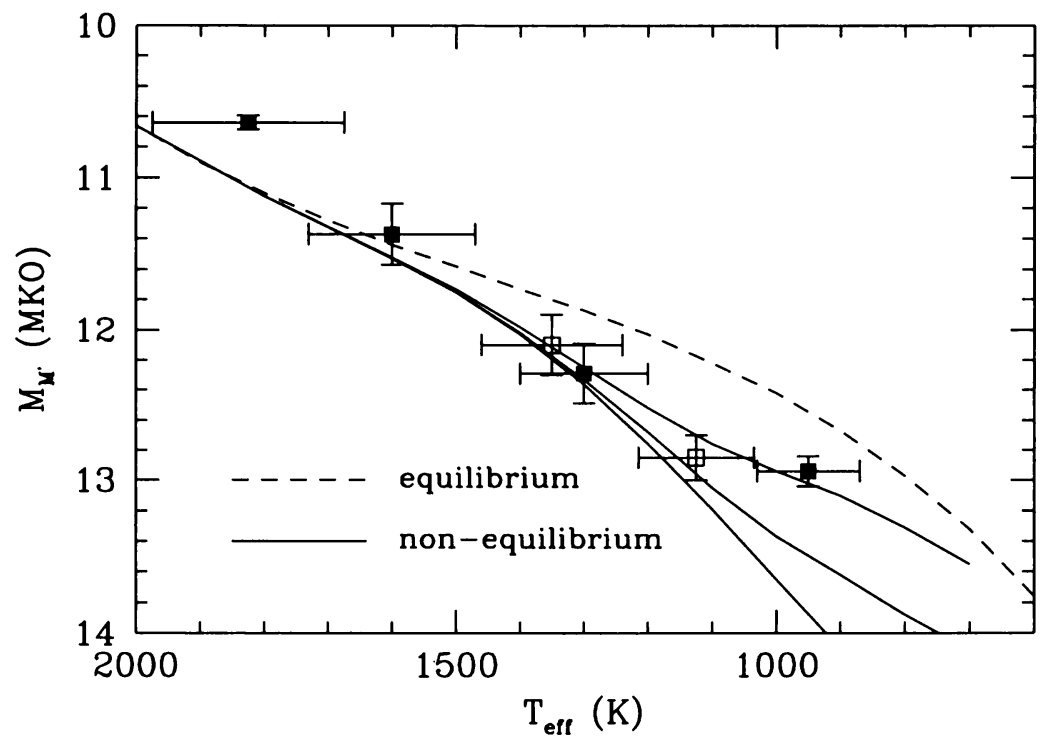

Figure 4. Absolute $M^{\prime}$ (MKO) magnitude versus effective temperature for L and T dwarfs. The data are from Leggett et al. (2002) and Golimowski et al. (2002). Open squares show 2MASS $0559-14$ as a single star (upper point) and as an equal pair binary (lower point). Curves show sequences of models with $g=10^{5} \mathrm{~cm} / \mathrm{s}^{2}$ for different values of the eddy diffusion coefficient. From top to bottom, $K=0,10^{2}$, $10^{4}$, and $10^{6} \mathrm{~cm}^{2} / \mathrm{s}$, respectively. $K=0$ corresponds to the equilibrium case.

\section{References}

Barshay, S. S. \& Lewis, J. S. 1978, Icarus, 33, 593

Burrows, A., Marley, M. S., Hubbard, W. B., Lunine, J. I., Guillot, T., Saumon, D., Freedman, R. S., Sudarsky, D., \& Sharp, C. 1997, ApJ, 491, 856

Fegley, B. Jr., \& Lodders, K. 1994, Icarus, 110, 117

Fegley, B. Jr., \& Lodders, K. 1996, ApJ, 472, L37

Fegley, B. Jr., \& Prinn, R. G. 1985, ApJ, 299, 1067

Golimowski, D. A., 2002, in preparation

Griffith, C. A. \& Yelle, R. V. 1999, ApJ, 519, L85

Kirkpatrick, J. D., Reid, I. N., Liebert, J., Cutri, R. M., Nelson, B., Beichman, C. A., Dahn, C. C., Monet, D. G., Gizis, J. E. \& Skrutskie, M. F. 1999, ApJ, 519, 834

Leggett, S. K. et al. 2002, ApJ, 564, 452

Leggett, S.K., Toomey, D.W., Geballe, T.R. \& Brown, R.H. 1999, ApJ, 517, L139

Lodders, K. \& Fegley, B. Jr. 1994, Icarus, 112, 368 
Lodders, K. \& Fegley, B. Jr. 2002, Icarus, 155, 393

Marley, M. S., Saumon, D., Guillot, T., Freedman, R. S., Hubbard, W. B., Burrows, A. \& Lunine, J. I. 1996, Science, 272, 1919

Nakajima, T., Oppenheimer, B. R., Kulkarni, S. R., Golimowski, D. A., Matthews, K., \& Durrance, S. T. 1995, Nature, 378, 463

Noll, K. S., Geballe, T. R. \& Marley, M. S. 1997, ApJ, 489, L87

Oppenheimer, B. R., Kulkarni, S. R., Matthews, K. \& Nakajima, T. 1995, Science, 270,1478

Oppenheimer, B. R., Kulkarni, S. R., Matthews, K. \& van Kerkwijk, M. H. 1998, ApJ, 502, 932

Prinn, R. G. \& Barshay, S. S. 1977, Science, 198, 1031

Saumon, D., Geballe, T. R., Leggett, S. K., Marley, M. S., Freedman, R. S., Lodders, K., Fegley, B. Jr. \& Sengupta, S. K. 2000, ApJ, 541, 374

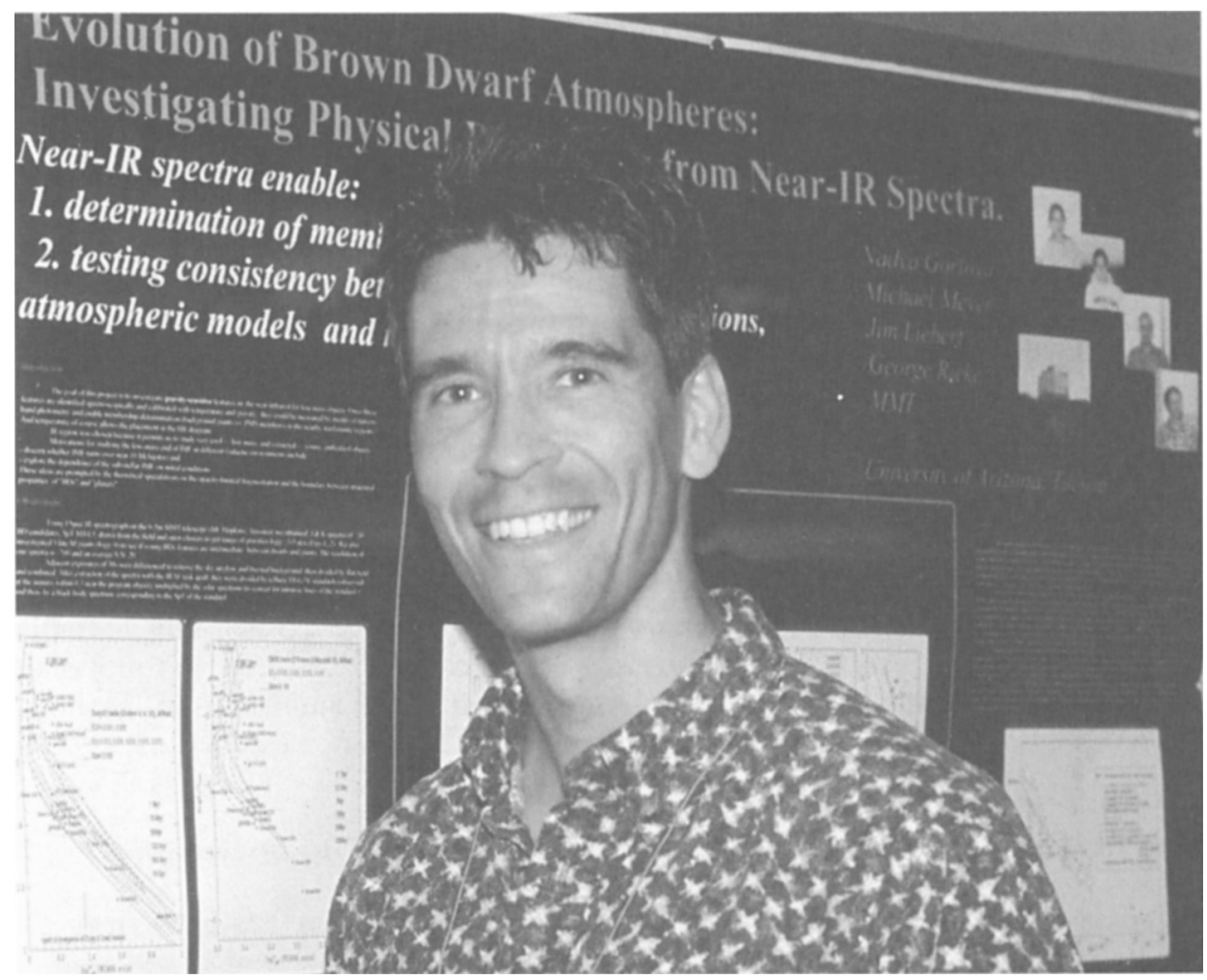

Didier Saumon 